\title{
The Necessary And Sufficient Condition For Consistency Of The MLE
}

\author{
QIQING YU \\ Department of Mathematical Sciences \\ State University of New York \\ Binghamton, NY 13902 \\ USA
}

https://www2.math.binghamton.edu/p/people/qyu/qyu_personal

\begin{abstract}
Suppose that the observations are i.i.d. from a density $f(; \theta)$, where $\theta$ is an identifiable parameter. One expects that the maximum likelihood estimator of $\theta$ is consistent. But its consistency proof is non-trivial and various sufficient conditions have been proposed (see, e.g., the classical statistics textbooks). All these sufficient conditions require $f(x ; \theta)$ being somewhat upper semi-continuous (in $\theta$ ), with various smoothness conditions or conditions needed for the dominated convergence theorem. We study the sufficient and necessary condition.
\end{abstract}

Key-Words: - Maximum likelihood estimator, consistency, Kullback-Leibler Inequality, parametric family, sufficient condition.

Received: January 7, 2021. Revised: May 8, 2021. Accepted: June 12, 2021. Published: July 2, 2021.

\section{Introduction.}

The maximum likelihood estimator (MLE) is a popular classical statistical method and is applied to many fields even recently (see, e.g., Lio and Liu [8] Derksen and Makam [4]). In this paper, the sufficient and necessary condition is studied for the strong consistency of MLE, assuming

(A1) $\mathbf{X}_{1}, \ldots, \mathbf{X}_{n}$ are i.i.d. from a random vector $\mathbf{X}$ with its density function (df) $f\left(\cdot ; \theta_{o}\right), \theta_{o} \in \Theta$;

(A2) the identifiability condition:

$\int\left|f(\mathbf{x} ; \theta)-f\left(\mathbf{x} ; \theta_{o}\right)\right| d \mu(\mathbf{x})=0$ implies $\theta=\theta_{o}$, where $\mu$ is a measure, $\theta$ is a finite dimensional parameter in $\Theta$ (the parameter space).

While (A1) is a common assumption of the MLE, (A2) is a necessary identifiability condition. If a parameter is not identifiable, we do not even know what is the true value of $\theta$, let alone a consistent estimator of $\theta$. Hereafter, we assume that (A1) and (A2) hold.

The new result is weaker than all the existing results in the literature (see, e.g., Ferguson ([5] Part 4), Lehmann and Casella ([7] Section 6.3), Stuart and Arnold ([12] Chapter 18), and Casella and Berger ([3] p.156)), among others), who only consider sufficient conditions, and is weaker than the results of Zhang [16] and Rossi [9] etc., who study also necessary conditions under additional assumptions rather than (A1) and (A2).

For illustration, consider first a continuous random variable (rv) $X$, with its $\mathrm{df} f$. There are 4 typical cases as follows:
Case 1. $\int f(t) \ln f(t) d t$ is finite, e.g., $f(t)=\mathbf{1}(t \in(0,1))$.

Case 2. $\int f(t) \ln f(t) d t=-\infty$, e.g., $f(t)=(r-1) \underset{t(\ln t)^{r}}{\mathbf{1}(t>e)}, r>1$.

Case 3. $\int f(t) \ln f(t) d t=\infty$, e.g.,

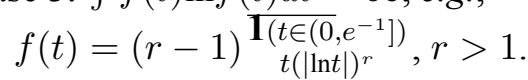

Case 4. $\int f(t) \ln f(t) d t$ does not exist, e.g., $f(t)=\frac{\mathbf{1}\left(t \in\left(0, e^{-1}\right] \cup[e, \infty)\right)}{2 t(\ln t)^{2}}$.

Each distribution in the examples of the four cases leads to a location or scale parameter family, among other possibilities. Thus we need to study the consistency of the MLE of the parameter in each case.

In many textbooks, people often say that the MLE is consistent under suitable conditions (see, e.g., Bickel and Doksum ([2] p.139.)). On the other hand, in his classical textbook, Ferguson ([5] p.114) shows that the MLE of $\theta$ is consistent if the following conditions hold:

(A3) $\lim _{\theta^{\prime} \rightarrow \theta} f\left(x ; \theta^{\prime}\right) \leq f(x ; \theta) \forall x$ (i.e., $f(\cdot ; \theta)$ is upper semi-continuous);

(A4) $\Theta$ is compact;

(A5) $\exists$ a function $K(x)$ such that $E_{\theta_{o}}(|K(X)|)<\infty$ and $\ln \underset{f\left(x ; \theta_{o}\right)}{f(x, \theta)} \leq K(x) \forall(x, \theta)$;

(A6) for all $\theta \in \Theta$, and sufficiently small $\delta>0$, $\sup _{\mid \theta-\overline{\theta_{o} \mid<\delta}} f(x ; \theta)$ is measurable in $x$.

(A5) is needed in his proof so that the dominated convergence theorem is applicable, but in the examples of Cases 2, 3 and 4, (A3), (A4) and (A5) do not hold (see Remark 1), not even in the examples of Case 1 with censored data (see Example 4). 
Casella and Berger ([3] p.516) present a set of somewhat simpler sufficient conditions for the consistency of the MLE of $\theta$ in their textbook as follows:

(A7) The densities $f(x ; \theta)$ have common support,

and $f(x ; \theta)$ is differentiable in $\theta$.

(A8) The parameter space $\Theta$ contains an open set $A$

and the true parameter $\theta_{o} \in A$.

Until recently (see, e.g., Rossi [9]) the sufficient conditions for the MLE under the assumptions of (A1) and (A2) are still essentially combinations of (A3), ..., (A8). Notice that (A7) implies (A3), and (A7) and (A8) are much stronger smoothness assumptions on the df. (A8) weaken (A4), but it does not allow discrete $\Theta$ and we may not know the true $A$, just like that we do not know the true value of $\theta_{0}$. One can verify that (A7) does not hold in the examples of Cases 1,2, 3 and 4 (see Remark 1).

It is actually more desirable to find the necessary and sufficient (NS) conditions for the consistency of the MLE. The NS conditions are studied for consistency of M-estimates in regression models with general errors (see Berlinet, Liese and Vajda [1]) or other models, but not for the MLE until recently. Zhang [16] establishes the NS condition for the weak consistency of the M-estimator under additional assumptions. In particular, Theorem 4 in Zhang [16] can be stated for the MLE $\hat{\theta}_{n}$ as follows:

(S1) Suppose that $X$ is a rv. $\rho_{n}(\theta)$ decreases with $\theta<\hat{\theta}_{n}$ and increases with $\theta>\hat{\theta}_{n}$, where $\rho_{n}(\theta)=$ $-\frac{1}{n} \sum_{i=1}^{n} \ln f\left(X_{i} ; \theta\right), \theta \in \mathcal{R}$. Then $\hat{\theta}_{n} \stackrel{P}{\rightarrow} \theta_{o}$ iff

$$
\begin{aligned}
& \inf _{\left\|\theta-\theta_{o}\right\| \geq \epsilon} \rho_{n}(\theta)-\rho_{n}\left(\theta_{o}\right) \stackrel{P}{\rightarrow} \delta(\epsilon) \forall \epsilon>0 \text { and } \\
& \text { a } \delta(\epsilon)>0 .
\end{aligned}
$$

Zhang's result has several drawbacks: (a) Eq. (1) is not always easy to check, (b) it is not a strong consistency result; (c) $X$ is not a random vector, (d) $\theta \in \mathcal{R}$, (e) convexity of $\mathcal{L}(\theta)$ in $\mathcal{R}$ may not be true (see Example 6).

Under (A1) and (A2), it is interesting to notice that almost all sufficient conditions in the literature imply that $f(\cdot ; \theta)$ is upper semi-continuous in $\theta$ (see, e.g., (A3) or (A7) (as continuity implies upper semicontinuity)), or its weakened version:

(A9) $\varlimsup_{n \rightarrow \infty} f\left(x ; \theta_{n}\right) \leq f\left(x ; \theta_{*}\right) \forall x \in \mathcal{W}$, where

$$
\int \mathbf{1}(x \notin \mathcal{W}) d \mu(x)=0 \text { and } \lim _{n \rightarrow \infty} \theta_{n}=\theta_{*}
$$

(see van der Vaart [13]). Under (A1) and (A2), in additional to upper semi-continuity (A3) or its weakened one (A9), all existing sufficient conditions in the literature need some additional regularity assumptions such as (A5), (A7), (A8) or (S1), among others. In this paper, it is shown that the sufficient condition for strong consistency of the MLE is only (A9) alone (see Theorems 1 and 2). The aforementioned parametric families all satisfy (A9) (see Section 4), but some of them do not satisfy (A3) and (A7) (see Remark 1). It is worth mentioning that since (A3) implies (A9), if (A3) holds then the MLE is consistent without (A4), (A5), (A7), (A8) and (S1), as imposed in the literature.

In Section 2, we present the sufficient condition for the strong consistency of the MLE when the observations are random variables (see Theorem 1 ). The extension of Theorem 1 to the case that the observations are $p \times 1$ random vectors is studied in Section 3 (see Theorem 2). In our proof of consistency, we need to modify the Kullback-Leibler (KL) [6] inequality. In Section 4, we establish the consistency of the MLEs of the parameters related to the examples in Cases 2, 3 and 4, as well as in Case 1 with censored data. We also explain that Theorem 2 can be applied to multivariate regression analysis. In Section 5, it is shown that (A9) is the necessary condition in some sense. Some proofs are relegated to a technical report for a better presentation.

\section{Main results for random variables.}

In order to motivate our main results, we shall first study some special cases. The examples in Cases 2, 3 and 4 can lead to parametric families that the existing sufficient conditions for the consistency of the MLE are not applicable. In some cases such as in Example 1 with complete data, the MLE has an explicit solution. Their consistency proofs are relatively easy in such cases, but not so otherwise.

Example 1. Consider an example in Case 4 with the df $f(x)=\left\{\begin{array}{ll}\frac{0.5}{(1+\ln x)^{2} x} & \text { if } x \geq 1 \\ \frac{0.5}{(1-\ln x)^{2} x} & \text { if } x \in(0,1)\end{array}\right.$ and $F(x)=P(X \leq x)= \begin{cases}1-\frac{0.5}{1+\ln x} & \text { if } x \geq 1 \\ \frac{0.5}{1-\ln x} & \text { if } x \in(0,1) .\end{cases}$ It leads to a location parameter family. Suppose that $X_{1}, \ldots, X_{n}$ are i.i.d. from $F(x-\alpha)$. The likelihood is $\mathcal{L}(\alpha)=0.5^{n} \times \prod_{i=1}^{n}\left\{\mathbf{1}\left(\alpha<X_{i}\right)\right.$

$$
\begin{aligned}
& \times\left[\left(1-\ln \left(X_{i}-\alpha\right)\right)^{2}\left(X_{i}-\alpha\right)\right]^{-\mathbf{1}\left(X_{i}-\alpha \in(0,1]\right)} \\
& \left.\left.\times\left[\left(1+\ln \left(X_{i}-\alpha\right)\right)^{2}\left(X_{i}-\alpha\right)\right)^{-\mathbf{1}\left(X_{i}-\alpha>1\right.}\right]\right\}, \text { i.e., } \\
& \qquad \mathcal{L}(\alpha) \begin{cases}=0 & \text { if } \alpha \geq X_{(1)} \\
=\infty & \text { if } \alpha=X_{(1)}- \\
<\infty & \text { if } \alpha<X_{(1)} .\end{cases}
\end{aligned}
$$

Thus the MLE under the location parameter family $F(x-\alpha)$ is $\hat{\alpha}=X_{(1)}$. It is well known that $P(\hat{\alpha}>t)=(P(X>t))^{n}$, thus the consistency follows, as well as the distribution of $\hat{\alpha}$. It is easy to show that $\hat{\alpha}$ is also strongly consistent.

The cumulative distribution function (cdf) $F(\cdot)$ in Example 1 also leads to a scale parameter family $\{F(\cdot / \theta): \theta>0\}$. The $\mathrm{df}$ is $f(x ; \theta)=\theta f(x / \theta)$

$$
=\frac{0.5}{\left[(1+\ln (x / \theta))^{2} x\right] \mathbf{1}_{(x>\theta)}\left[(1-\ln (x / \theta))^{2} x\right]} \mathbf{1}_{(x \in(0, \theta])}
$$


$=\frac{0.5}{x(1+\ln (x / \theta))^{2} \mathbf{\mathbf { 1 }}_{(x>\theta>0)}(1-\ln (x / \theta))^{2} \mathbf{\mathbf { 1 }}_{(x \in(0, \theta])}}, x>0$.

Thus for $X_{(1)}>0, \mathcal{L}(\theta)=\prod_{i=1}^{n} f\left(x_{i} ; \theta\right)$

$=\frac{0.5^{n} / \prod_{i} X_{i}}{\prod_{i}\left(1+\ln \left(X_{(i)} / \theta\right)\right)^{2} \mathbf{1}_{\left(X_{(i)}>\theta>0\right)}\left(1-\ln \left(X_{(i)} / \theta\right)\right)^{2} \mathbf{1}_{\left(0<X_{(i)} \leq \theta\right)}}$,

$g(\ln \theta) \stackrel{\text { def }}{=}\left(\ln \mathcal{L}(\theta)+\sum_{i} \ln X_{i}+n \ln 2\right) / 2$

$=-\sum_{i>k}^{n} \ln \left(1+\ln \frac{X_{(i)}}{\theta}\right)-\sum_{i=1}^{k} \ln \left(1-\ln \frac{X_{(i)}}{\theta}\right)$,

if $X_{(k)} \leq \theta<X_{(k+1)}, 0 \leq k \leq n$.

$g(\ln \theta)=\left\{\begin{array}{cl}-\infty & \text { if } \theta=0+\text { or } \infty \\ -\sum_{i=1}^{n} \ln \left(1+\ln \frac{X_{(i)}}{\theta}\right) & \text { if } 0<\theta<X_{(1)} \\ -\sum_{i=1}^{n} \ln \left(1-\ln \frac{X_{(i)}}{\theta}\right) & \text { if } X_{(n)} \leq \theta \\ -\left[\sum_{i>k}^{n} \ln \left(1+\ln \frac{X_{(i)}}{\theta}\right)\right. & \\ \left.\quad+\sum_{i=1}^{k} \ln \left(1-\ln \frac{X_{(i)}}{\theta}\right)\right] & \text { otherwise, }\end{array}\right.$

where $X_{(k)} \leq \theta<X_{(k+1)}, 1 \leq k<n$.

Let $h(x)=\ln (a \pm x)$, then $h^{\prime}= \pm(a \pm x)^{-1}$ and $h^{\prime \prime}(x)=(a \pm x)^{-2}$. Thus, if $t \neq \ln X_{(k)}, k=1, \ldots, n$, then $g^{\prime}(t)=\sum_{i} \frac{\mathbf{1}\left(X_{(i)}>e^{t}\right)}{1+\ln X_{(i)}-t}-\sum_{i} \frac{\mathbf{1}\left(X_{(i)} \leq e^{t}\right)}{1-\ln X_{(i)}+t}$,

$$
g^{\prime \prime}(t)=\sum_{i} \frac{\mathbf{1}\left(X_{(i)}>e^{t}\right)}{\left(1+\ln X_{(i)}-t\right)^{2}}+\sum_{i} \frac{\mathbf{1}\left(X_{(i)} \leq e^{t}\right)}{\left(1-\ln X_{(i)}+t\right)^{2}} .
$$

Hence, $g^{\prime}(\ln \theta)$ is a monotonely increasing function of $\ln \theta$ for $\theta \in\left(X_{(i)}, X_{(i+1)}\right), i \in\{0,1, \ldots, n\}$. For example, consider a special case by letting $n=2$, $X_{1}=1$ and $X_{2}=e$. Then

$$
g(t)=-\mathbf{1}(0>t) \ln (1-t)-\mathbf{1}(0 \leq t) \ln (1+t)-
$$
$\mathbf{1}(1>t) \ln (2-t)-\mathbf{1}(1 \leq t) \ln (t)$.

$$
g(t)= \begin{cases}-\mathbf{1}(0>t) \ln (1-t) & \\ \mathbf{- 1}(1>t) \ln (2-t)=-\infty & \text { if } t=-\infty \\ \mathbf{- 1}(0>t) \ln (1-t) & \\ \mathbf{- 1}(1>t) \ln (2-t)=-\ln 2 & \text { if } t=0- \\ \mathbf{- 1}(0 \leq t) \ln (1+t) & \\ \mathbf{- 1}(1>t) \ln (2-t)=-\ln 2 & \text { if } t=0+ \\ \mathbf{- 1}(0 \leq t) \ln (1+t) & \\ \mathbf{- 1}(1>t) \ln (2-t)=-\ln 2.25 & \text { if } t=0.5 \\ \mathbf{- 1}(0 \leq t) \ln (1+t) & \text { if } t=1- \\ \mathbf{- 1}(1>t) \ln (2-t)=-\ln 2 & \text { if } t=1+ \\ \mathbf{- 1}(0 \leq t) \ln (1+t) & \text { if } t=\infty \\ \mathbf{- 1}(1 \leq t) \ln (t)=-\ln 2 & \end{cases}
$$$$
g^{\prime}(t)= \begin{cases}-\frac{\mathbf{1}(0 \leq t)}{1+t}-\frac{\mathbf{1}(1 \leq t)}{t}=0- & \text { if } t=\infty \\ -\frac{\mathbf{1}(0 \leq t)}{1+t}-\frac{\mathbf{1}(1 \leq t)}{t}<0 & \text { if } t=1+ \\ -\frac{\mathbf{1}(0 \leq t)}{1+t}+\frac{\mathbf{1}(1>t)}{2-t}>0 & \text { if } t=1- \\ -\frac{\mathbf{1}(0 \leq t)}{1+t}+\frac{\mathbf{1}(1>t)}{2-t}<0 & \text { if } t=0+ \\ \frac{\mathbf{1}(0>t)}{1-t}+\frac{\mathbf{1}(1>t)}{2-t}>0 & \text { if } t=0- \\ \frac{\mathbf{1}(0>t)}{1-t}+\frac{\mathbf{1}(1>t)}{2-t}=0+ & \text { if } t=-\infty\end{cases}
$$

$\begin{array}{cccccccc}t: & -\infty & & 0- & 0+ & & & 1- \\ g^{\prime}(t): & 0+ & \nearrow & 1.5 & -0.5 & \nearrow & \nearrow & +0.5 \\ g(t): & -\infty & \nearrow & -\ln 2 & -\ln 2 \\ t: & 1+ & & \infty & & & \nearrow & -\ln 2 \\ g^{\prime}(t): & -1.5 & \nearrow & 0- & & & & \\ g(t): & -\ln 2 & \searrow & -\infty & & & & \end{array}$

Notice that

(a) $\mathcal{L}(\theta)$ is a continuous function in $\theta$;

(b) $\mathcal{L}(\theta)$ is twice differentiable in the intervals $\left(X_{(i-1)}, X_{(i)}\right), i=0, \ldots, n$;

(c) but $\mathcal{L}(\theta)$ is not differentiable at $X_{(i)}$ 's;

(d) $g^{\prime \prime}(t)>0$ for $t \in\left(e^{X_{(i-1)}}, e^{X_{(i)}}\right), i=0, \ldots, n$;

(e) thus $g^{\prime}(t)$ is strictly increasing for $t \in\left(e^{X_{(i-1)}}, e^{X_{(i)}}\right), i=0, \ldots, n$

(f) thus $g^{\prime}(t)=0$ at the local minimum points.

The MLE $\hat{\theta}_{n}=\operatorname{argmax}_{t \in\left\{X_{1}, \ldots, X_{n}\right\}} g(\ln t)$, where $g\left(\ln X_{(k)}\right)=\sum_{i>k}^{n}\left(1+\ln \left(X_{(i)} / X_{(k)}\right)\right)^{-1}$

$-\sum_{i<k}\left(1-\ln \left(X_{(i)} / X_{(k)}\right)\right)^{-1}, k=0, \ldots, n$.

The examples in Cases 2, 3 and 4 can also lead to certain location or scale parameter families. The existing sufficient conditions for the consistency of the MLE are not applicable (see Remark 1).

\section{The example in Case 2.}

$f(t)=(r-1) \mathbf{1}(t>e) /\left[t(\ln t)^{r}\right], r>1$.

The location parameter:

$f(t ; \alpha)=(r-1) \mathbf{1}(t-e>\alpha) /\left[(t-\alpha)(\ln (t-\alpha))^{r}\right]$. $f(t ; \alpha) \uparrow$ in $\alpha \in(-\infty, t-e]$, as $\frac{1}{t-\alpha} \uparrow$ and $\frac{1}{(\ln (t-\alpha))^{r}} \uparrow$. Thus $\mathcal{L}(\alpha) \uparrow$ in $\alpha \in\left(-\infty, X_{(1)}-e\right]$, the MLE of $\alpha$ is $\hat{\alpha}_{n}=X_{(1)}-e$.

The scale parameter:

$f(t ; \theta)=(r-1) \mathbf{1}(t>e \theta) /\left[t(\ln (t / \theta))^{r}\right], r>1$.

Thus the MLE of $\theta$ is $\hat{\theta}_{n}=X_{(1)} / e$.

The example in Case 3.

$f(t)=(r-1) \mathbf{1}(t \in(0,1 / e]) /\left[t(\ln t)^{r}\right], r>1$.

The location parameter:

$f(t ; \alpha)=\frac{(r-1) \mathbf{1}(t \in[\alpha, \alpha+1 / e])}{(t-\alpha)(\ln (t-\alpha))^{r}}, r>1$.

$\mathcal{L}(\alpha)=\frac{\left.\mathbf{1}\left(\alpha<X_{(1)} \leq \cdots \leq X_{(n)}<\alpha+1 / e\right]\right)}{\prod_{i=1}^{n}(r-1)\left[\left(X_{i}-\alpha\right)\left(\ln \left(X_{i}-\alpha\right)\right)^{r}\right]}$

$\mathcal{L}(\alpha)=\uparrow$ in $\alpha \in\left[X_{(n)}-1 / e, X_{(1)}\right]$.

Thus the MLE of $\alpha$ is $\hat{\alpha}_{n}=X_{(1)}$.

The scale parameter:

$f(t ; \theta)=\frac{(r-1) \mathbf{1}(t \in(0, \theta / e))}{t(\ln (t / \theta))^{r}}, r>1$.

Thus the MLE of $\theta$ is $\hat{\theta}_{n}=X_{(n)} e$

An example in Case 4. Let $u, v>1, p \in[0,1]$, $f(t)=p \frac{(v-1) \mathbf{1}(t \in(0,1])}{(1-\ln t)^{v} t}+q \frac{(u-1) \mathbf{1}(t>1)}{(1+\ln t)^{u} t}$. Then

$F(t)=\frac{p \mathbf{1}(t \in(0,1))}{(1-\ln t)^{v}}+q\left[1-\frac{\mathbf{1}(t \geq 1)}{(1-\ln t)^{u}}\right], q=1-p$. 
The location parameter $\alpha$ :

$f(t ; \alpha)=\frac{p(v-1) \mathbf{1}(t-a \in(0,1])}{(1-\ln (t-a))^{v}(t-a)}+\frac{q(u-1) \mathbf{1}(t-\alpha>1)}{(1+\ln (t-a))^{u}(t-a)}$. Thus $\alpha<X_{(1)}$. Since $\mathcal{L}(\alpha)\left\{\begin{array}{ll}=\infty & \text { if } \alpha=X_{(1)}- \\ <\infty & \text { otherwise, }\end{array}\right.$ the MLE is $\hat{\alpha}_{n}=X_{(1)}$.

The scale parameter $\theta$ :

$f(t ; \theta)=p \frac{(v-1) \mathbf{1}(t / \theta \in(0,1])}{(1-\ln (t / \theta))^{v} t}+q \frac{(u-1) \mathbf{1}(t / \theta>1)}{(1+\ln (t / \theta))^{u} t}$. Since $\ln f=-\ln t+$

$$
\begin{aligned}
& \mathbf{1}(t \in(0, \theta))[\ln (p(v-1))-v \ln (1-\ln t+\ln \theta)] \\
& \quad+\mathbf{1}(t>\theta)[\ln (q(u-1))-u \ln (1+\ln t-\ln \theta)],
\end{aligned}
$$$$
g(\ln \theta)
$$$$
\stackrel{\text { def }}{=} \ln \mathcal{L}(\theta)+\sum_{i} \ln X_{i}-\sum_{\left.X_{i} \in(0, \theta]\right)} \ln (p(v-1))
$$$$
-\sum_{X_{i}>\theta} \ln (q(u-1))
$$$$
\begin{aligned}
& -\sum_{X_{i}}>\theta \ln (q(u-1)) \\
= & -\sum_{i} \mathbf{1}\left(X_{i} \in(0, \theta]\right) v \ln (1-\ln t+\ln \theta)
\end{aligned}
$$$$
\left.-\sum_{i} \mathbf{1}\left(X_{i}>\theta\right)\right) u \ln (1+\ln t-\ln \theta)
$$$$
=-u \sum_{i>k}^{n} \ln \left(1+\ln X_{(i)}-\ln \theta\right)
$$

$-v \sum_{i>1}^{k} \ln \left(1-\ln X_{(i)}+\ln \theta\right)$, where

$X_{(k)} \leq \theta \stackrel{<}{<} X_{(k+1)}, k=0,1, \ldots, n$ and $X_{(0)}=0$.

Notice that $\frac{d}{d \theta} g(\ln \theta)$ does not exist at $\theta=X_{i} \forall i$, and $g^{\prime \prime}(t)=\sum_{i>k} \frac{u}{\left(1+\ln X_{(i)}-t\right)^{2}}+\sum_{i=1}^{k} \frac{v}{\left(1-\ln X_{(i)}+t\right)^{2}}>0$ if it exists. Thus $\hat{\theta}_{n}=\operatorname{argmax}_{\theta \in\left\{X_{(1)}, \ldots, X_{(n)}\right\}} \mathcal{L}(\theta)$. It worth mentioning that the Newton-Raphson algorithm does not work here.

In addition to $(\alpha, \theta), u, v$ and $p$ can also be treated as parameters. One can find the MLE of $u$ :

$\ln f \propto \ln (u-1)-u \ln (1+\ln t)$.

$\frac{d}{d u} \sum_{i} \ln f\left(X_{i} ; u\right)=\sum_{X_{i}>1}\left[\frac{1}{u-1}-\ln \left(1+\ln X_{i}\right)\right]=0$ yields $\sum_{X_{i}>1} \frac{1}{u-1}=\sum_{X_{i}>1} \ln \left(1+\ln X_{i}\right)$.

Thus the MLE $\hat{u}=1+\frac{\sum_{X_{i}>1} 1}{\sum_{X_{i}>1} \ln \left(1+\ln X_{i}\right)}$,

$$
\text { as } \frac{d^{2}}{d u^{2}} \sum_{i} \ln f\left(X_{i} ; u\right)=-\sum_{X_{i}>1} \frac{1}{(u-1)^{2}}<0 .
$$

One can also find the MLE of $p$ and $v$.

Remark 1. Condition (A7) in Casella and Berger [3] and conditions (A3), (A4), (A5) in Ferguson [5] are violated if $f(x ; \theta, \alpha)=\frac{\mathbf{1}(x \in(\alpha, \alpha+\theta / e])}{(x-\alpha)(\ln ((x-\alpha) / \theta))^{2}}$ (an example of Case 3 ).

(A7) fails: $f(x ; \theta, \alpha)$ is not continuous in $\theta$ at $\theta=\theta_{*}$ for $x \in\left\{\alpha, \alpha+\theta_{*} / e\right\}$ and for each $\theta_{*}>0$, due to the factor $\mathbf{1}(x \in(\alpha, \alpha+\theta / e])$ in $f(x ; \theta, \alpha)$. The support of $f(x ; \theta, \alpha)$ is $\begin{cases}{[0,1 / e]} & \text { if } \theta=1 \text { and } \alpha=0 \\ {[0,1 /(2 e)]} & \text { if } \theta=0.5 \text { and } \alpha=0\end{cases}$ (not the same).

(A3) fails: $\varlimsup_{n \rightarrow \infty} f\left(x ; \theta_{n}, \alpha_{n}\right)=\infty(\not \leq f(x ; 1,0)=$ 0 ) if $\theta_{n}=1$ and $\alpha_{n}=(-1)^{n} / n+x$.

(A4) fails: $\Theta$ is not compact, as $\Theta=(0, \infty) \times$ $(-\infty, \infty) \neq \bar{\Theta}$ (the closure of $\Theta$ ).

(A5) fails: If $\left(\theta_{o}, \alpha_{o}\right)=(1,0)$ and $\theta \geq 1$, then

$$
\begin{aligned}
& \frac{f(x ; \theta, \alpha)}{f\left(x ; \theta_{o}, \alpha_{o}\right)} \\
= & \frac{\mathbf{1}(x-\alpha \in(0, \theta / e]) x(\ln x)^{2}}{(x-\alpha)(\ln ((x-\alpha) e / \theta))^{2} \mathbf{1}(x \in(0,1 / e])}
\end{aligned}
$$

$=\infty$, if $x=\alpha+>\alpha_{o}$ and $x<1 / e$. If (A5) were true, then $\exists$ a function $K(x)$ such that

$$
\begin{aligned}
& E_{\theta_{o}, \alpha_{o}}(|K(X)|)<\infty \text { and } \\
& K(x) \geq \ln \frac{f(x ; \theta ; \alpha)}{f\left(x ; \theta_{o}, \alpha_{o}\right)} \forall(x, \theta, \alpha),
\end{aligned}
$$

then $K(x) \geq \ln \frac{f(x ; \theta, \alpha)}{f\left(x ; \theta_{o}, \alpha_{o}\right)}=\ln \infty \forall x=\alpha+>\alpha_{o}=$ 0 and $x<1 / e$ by Eq.(2), i.e. $K(x)=\infty$ for all $x \in(0,1 / e]$ and $\int_{0}^{1 / e} f_{o}\left(x ; \theta_{o}\right) d x=1$. Consequently $\infty>E_{\theta_{o}, \alpha_{o}}(|K(X)|)=E(\infty)=\infty$, a contradiction. $\square$

Since the existing results on the sufficient conditions of the consistency of the MLE are not applicable to the families of distributions in Cases 2, 3 and 4, we propose a weaker sufficient condition for consistency of the MLE in Theorem 1. The consistency of the MLEs in the examples in Cases 2, 3 and 4 can be proved by verifying the sufficient condition proposed in Theorem 1. This is done in Section 4.

Before we present the main theorem, we shall present some preliminary results. We shall make use of the following inequality:

KL inequality. $K \stackrel{\text { def }}{=} \int f_{o}(t) \ln \left(f_{o} / f\right)(t) d \mu(t) \geq 0$; with equality iff $\int\left|f(t)-f_{o}(t)\right| d \mu(t)=0$.

Kullback and Leibler [6] show that $K$ exists, though it is possible that $K=\infty$.

The KL inequality requires that $f$ and $f_{o}$ are both densities w.r.t. the measure $\mu$. That is, $\int f_{o} d \mu=$ $\int f d \mu=1$. However, we encounter the case $\int f d \mu<1$ in our proof such as in Example 2.

Example 2. Let $f(x ; \theta)=\mathbf{1}(x \in(0, \theta]) / \theta, \theta>0$. $F(x ; 0)=\lim _{\theta \downarrow 0} F(x ; \theta)=\mathbf{1}(x \geq 0) \forall x . f(\cdot ; 0)$ is a point mass at 0 and $\int f(x ; 0) d x=0<1=$ $\int f(x ; \theta) d x$ if $\theta>0$.

We thus modify the KL inequality as follows.

Lemma 1 (Yu [14]). If $f \geq 0, f_{o} \geq 0, \mu$ is a measure, $\int f_{o}(t) d \mu(t)=1$ and $\int f(t) d \mu(t) \leq 1$, then $\int f_{o}(t) \ln \frac{f_{o}(t)}{f(t)} d \mu(t) \geq 0$, with equality iff $f=f_{o}$ a.e. w.r.t. $\mu$.

We would also make use of Fatou's Lemma with varying measures as follows.

Lemma 2 (Propositions 17 and 18 in Royden ([10] page 231)). Let $(\mathcal{S}, \mathcal{B})$ be a measurable space, $\left\{\mu_{n}\right\}_{n \geq 1}$ a sequence of measures which converge setwise to a measure $\mu$ (l.e., $\lim _{n \rightarrow \infty} \mu_{n}(B)=\mu(B), \forall$ $B \in \mathcal{B}), g_{n}$ and $f_{n}$ are non-negative measurable functions, and $\left(f_{n}, g_{n}\right)(x)$ converges pointwise to the vector of functions $(f, g)(x)$. We have

(a) $\int f d \mu \leq \lim _{n \rightarrow \infty} \int f_{n} d \mu_{n}$; 
(b) if $f_{n} \leq g_{n}$ and $\lim _{n \rightarrow \infty} \int g_{n} d \mu_{n}=\int g d \mu$, then $\int f d \mu=\lim _{n \rightarrow \infty} \int f_{n} d \mu_{n}$.

Corollary 1. Suppose that $\mu_{n}$ is a sequence of measures on the measurable space $(\mathcal{S}, \mathcal{B})$ such that $\lim _{n \rightarrow \infty} \mu_{n}(B) \rightarrow \mu(B), \forall B \in \mathcal{B}, f$ and $f_{n}$ are integrable functions, $n \geq 1$.

(a) If $f_{n}$ are bounded below and $f(x)=\lim _{n \rightarrow \infty} f_{n}(x)$, then $\int f d \mu \leq \lim _{n \rightarrow \infty} \int f_{n} d \mu_{n}$.

(b) If $f_{n}$ are bounded below then $\int \varliminf_{n \rightarrow \infty} f_{n} d \mu \leq \varliminf_{n \rightarrow \infty} \int f_{n} d \mu_{n}$.

Proof. Statement (a) is the same as Corollary $1 \mathrm{Yu}$ [14]. We now prove statement (b):

Let $g_{n}(x)=\inf \left\{f_{k}(x): k \geq n\right\}$, then $g_{n}(x) \rightarrow$ $g(x)=\varliminf_{n \rightarrow \infty} f_{n}(x)$. We have

$\int \lim _{n \rightarrow \infty} f_{n} d \mu=\int \lim _{n \rightarrow \infty} g_{n} d \mu$

$\leq \varliminf_{n \rightarrow \infty} \int g_{n} d \mu_{n}$ (by statement (a),

as $g_{n}$ is bounded below)

$=\varliminf_{n \rightarrow \infty} \int \inf \left\{f_{k}: k \geq n\right\} d \mu_{n}$

$\leq \lim _{n \rightarrow \infty} \int f_{n} d \mu_{n}$.

Lemma 3. Given each sequence of cdf's $\left\{F\left(\cdot ; \theta_{n}\right)\right\}_{n \geq 1}, \quad \exists a$ pointwise convergence subsequence $\left\{F\left(\cdot ; \theta_{n_{j}}\right\}_{j \geq 1}\right.$ with the limit function $F(\cdot) \in \mathcal{F}$, where $\mathcal{F}$ is a collection of all $F$ satisfying that $F(x)$ is a nondecreasing function on $[-\infty, \infty]$, $F(-\infty)=0$ and $F(\infty)=1$.

Here $\mathcal{F}$ includes $F$ satisfying $\lim _{x \rightarrow \infty} F(x)<1$, or $\lim _{x \rightarrow-\infty} F(x)>F(-\infty)=0$, or $\lim _{t \downarrow x} F(t)>$ $F(x)$. Lemma 3 is a trivial special case of Helly's selection theorem (Rudin [11] p.167), thus its proof is skipped. $F(x ; \theta)=\int \mathbf{1}(t \leq x) f(t ; \theta) d \mu(t)$ if $\theta \in \Theta$. If $\theta_{n} \in \Theta$ and $\theta_{n} \rightarrow \theta_{*} \notin \Theta$, then there is a convergent sub-sequence of $\left\{F\left(\cdot ; \theta_{n}\right)\right\}_{n \geq 1}$ by Lemma 3 , say $F\left(\cdot ; \theta_{j_{n}}\right) \rightarrow F\left(\cdot ; \theta_{*}\right)$. Then $f\left(x ; \theta_{*}\right)$ needs to be defined. For technical reasons in the proof of consistency, define

$$
f\left(x ; \theta_{*}\right)=\varlimsup_{n \rightarrow \infty} f\left(x ; \theta_{j_{n}}\right) .
$$

Remark 2. It is worth mentioning that $f\left(\cdot ; \theta_{*}\right)$ defined in $E q$. (3) may not be a df w.r.t. $\mu$. If $\theta_{*} \notin \Theta$ then we may not have

$$
F\left(x ; \theta_{*}\right)=\int \mathbf{1}(t \leq x) f\left(t ; \theta_{*}\right) d \mu(t) .
$$

For instance, let $f_{n}(x)=\mathbf{1}(x \in(0,1 / n)) n$ as in Example 2, where $\theta_{n}=1 / n \rightarrow 0=\theta_{*}$ and $\mu$ is the Lebesgue measure. Then

$F_{n}(x)=\int \mathbf{1}(t \leq x) f_{n}(t) d t \rightarrow \mathbf{1}(x \geq 0) \stackrel{\text { def }}{=} F\left(x ; \theta_{*}\right)$. Eq. (3) yields

$f\left(x ; \theta_{*}\right)=\lim _{n \rightarrow \infty} f_{n}(x)=0$ (not a df w.r.t. $\left.\mu\right)$.
Lemma 4. If (A9) holds and if $\exists\left\{\theta_{n}\right\}_{n \geq 1} \subset \Theta$ such that $\theta_{n} \rightarrow \theta_{*}$ and $\lim _{n \rightarrow \infty} F\left(x ; \theta_{n}\right)$ exists $\forall x$, then by defining $f\left(\cdot ; \theta_{*}\right) \stackrel{\text { def }}{=} \varlimsup_{n \rightarrow \infty}^{n \rightarrow \infty} f\left(\cdot ; \theta_{n}\right)$ if $\theta_{*} \notin \Theta$, we have

$$
\varlimsup_{n \rightarrow \infty} f\left(x ; \theta_{n}\right) \leq f\left(x ; \theta_{*}\right) \text { if } x \in \mathcal{W},
$$

where $\int \mathbf{1}(x \notin \mathcal{W}) d \mu(x)=0$.

Proof. Assume (A9) holds. If $\theta_{*} \in \Theta$, Eq.(4) follows from (A9). Otherwise, Eq.(4) follows from $f\left(\cdot ; \theta_{*}\right) \stackrel{\text { def }}{=} \varlimsup_{n \rightarrow \infty} f\left(\cdot ; \theta_{n}\right)$ if $\theta_{*} \notin \Theta$. .

Remark 3. (A9) is a weakened version of (A3) (the upper semi-continuity) in three senses:

(a) It does not require $\varlimsup_{n \rightarrow \infty} f\left(x ; \theta_{n}\right) \leq f\left(x ; \theta_{*}\right)$ for all $x$;

(b) It allows $\Theta$ be a nowhere-dense set;

(c) It yields Eq.(4), which does not require $\theta_{*} \in \Theta$, if $\Theta$ is not compact (i.e. (A4) does not hold).

Remark 4. (A9) weakens (A7) too: (a) (A9) is implied by the existence of $\frac{\partial f(\cdot ; \theta)}{\partial \theta}$ in (A7); (b) (A9) does not require that the densities $f(x ; \theta)$ have common support as in (A7).

Remark 5. For technical reason, we define the $M L E$ $\hat{\theta}=\hat{\theta}_{n}=\operatorname{argmax}_{\theta \in \bar{\Theta}} \prod_{i=1}^{n} f\left(X_{i} ; \theta\right)$, rather than $\hat{\theta}=\operatorname{argmax}_{\theta \in \Theta} \prod_{i=1}^{n} f\left(X_{i} ; \theta\right)$. Hereafter, let $\hat{F}_{n}$ be the empirical distribution function (edf) based on $X_{i}$ 's and $\Omega_{o}$ the subset of the sample space $\Omega$ such that $\hat{F}_{n}(x) \rightarrow F\left(x ; \theta_{o}\right) \forall x$, where $F\left(\cdot ; \theta_{o}\right)=F_{X}(\cdot)$.

We shall make use of the following condition.

(A10) $\varlimsup_{n \rightarrow \infty} f\left(x ; \hat{\theta}_{j_{n}}\right)(\omega) \leq f\left(x ; \theta_{*}(\omega)\right) \forall x \in \mathcal{W}$,

$\forall \omega \in \Omega_{o}$ and $\forall$ convergent subsequence

$\left\{\hat{\theta}_{j_{n}}\right\}_{n \geq 1}$ of the MLE $\hat{\theta}_{n}$, where $\hat{\theta}_{j_{n}}(\omega) \rightarrow \theta_{*}(\omega)$ and $\int \mathbf{1}(x \notin \mathcal{W}) d \mu(x)=0$.

Theorem 1. Suppose that $X_{i}$ 's are random variables and (A1) and (A2) hold. Then the MLE $\hat{\theta} \stackrel{\text { a.s. }}{\rightarrow} \theta_{O}$ and $f\left(x ; \hat{\theta}_{n}\right) \stackrel{\text { a.s. }}{\rightarrow} f\left(x ; \theta_{0}\right) \forall x \in \mathcal{W}$, if (A9) or (A10) holds.

Proof. By assumptions (A1) and (A2), $P\left(\Omega_{0}\right)=1$. For each $\omega \in \Omega_{o}$, let $\theta_{*}$ be a limiting point of $\hat{\theta}_{n}(\omega)$, where $\theta_{*} \in \bar{\Theta}$. Then the MLE $\hat{\theta}_{n}$ is consistent iff $\theta_{*}=\theta_{o}$, Thus the theorem is proved once we prove $\theta_{*}=\theta_{o}$, which is done next.

Hereafter, fixed $\omega \in \Omega_{o}$. Then there exists a convergent subsequence of $\left\{F\left(\cdot ; \hat{\theta}_{n}\right)\right\}_{n \geq 1}$ by Lemma 3. By taking a convergence subsequences of $\left\{\hat{\theta}_{n}\right\}_{n \geq 1}$ and $\left\{F\left(\cdot ; \hat{\theta}_{n}\right)\right\}_{n \geq 1}$, without loss of generality (WLOG), we can assume $\hat{\theta}_{n} \rightarrow \theta_{*}$ and $\hat{F}\left(\cdot, \hat{\theta}_{n}\right)$ converges to $F\left(\cdot ; \theta_{*}\right)(\in \mathcal{F}$ (see Lemma 3$)$ ) pointwisely. Thus (A9) and Lemma 4 yield Eq.(4), with $\theta_{n}=\hat{\theta}_{n}$, i.e.,

$$
\varlimsup_{n \rightarrow \infty} f\left(x ; \hat{\theta}_{n}\right) \leq f\left(x ; \theta_{*}\right), x \in \mathcal{W}
$$


where $\int \mathbf{1}(x \notin \mathcal{W}) d \mu(x)=0$. On the other hand, Eq.(5) follows from (A10) directly.

The normalized log-likelihood is $\sum_{i=1}^{n} \ln f\left(X_{i} ; \theta\right) / n$. The MLE $\hat{\theta}_{n}$ yields,

$$
\begin{aligned}
0 & \geq \frac{1}{n} \sum_{i=1}^{n} \ln \frac{f\left(X_{i} ; \theta_{o}\right)}{f\left(X_{i} ; \hat{\theta}_{n}\right)}=\int \ln \frac{f\left(t ; \theta_{o}\right)}{f\left(t ; \hat{\theta}_{n}\right)} d \hat{F}_{n}(t) \\
& =\int H\left(\frac{f\left(t ; \theta_{o}\right)}{f\left(t ; \hat{\theta}_{n}\right)}\right) \frac{f\left(t ; \hat{\theta}_{n}\right)}{f\left(t ; \theta_{o}\right)} d \hat{F}_{n}(t) \quad(H(t) \stackrel{d e f}{=} t \ln t) .
\end{aligned}
$$

Denote $A_{k}=\left\{t: \frac{f\left(t ; \hat{\theta}_{n}\right)}{f\left(t ; \theta_{o}\right)} \leq k, \forall n\right\}$ and $B_{k}=A_{k} \backslash A_{k-1}, k \geq 1$. Notice that $\frac{f\left(t ; \hat{\theta}_{n}\right)}{f\left(t ; \theta_{o}\right)}$ is finite for each $n$, provided that

$t \in\left\{x:\left|F\left(x+s ; \theta_{o}\right)-F\left(x ; \theta_{o}\right)\right|>0 \forall s \neq 0\right\}$. Then

$$
\int \mathbf{1}\left(\cup_{k \geq 1} B_{k}\right) d F\left(t ; \theta_{o}\right)=1 .
$$

For each $k \geq 1$, let $a_{k}=\mathbf{1}\left(t \in B_{k}\right) \ln \left(\frac{f\left(t ; \theta_{o}\right)}{f\left(t ; \hat{\theta}_{n}\right)}\right)$ and define $\ln 0=-\infty$. We have

$$
\varliminf_{n \rightarrow \infty} \int_{B_{k}} \ln \frac{f\left(t ; \theta_{o}\right)}{f\left(t ; \hat{\theta}_{n}\right)} d \hat{F}_{n}(t)
$$

$\geq \int_{B_{k}} \varliminf_{n \rightarrow \infty} \ln \frac{f\left(t ; \theta_{o}\right)}{f\left(t ; \hat{\theta}_{n}\right)} d F\left(t ; \theta_{o}\right) \quad$ (by (b) of Corollary 1 as $\left.a_{k} \in[-\ln k,-\ln (k-1)], k \geq 1\right)$

$=\int_{B_{k}} \ln \lim _{n \rightarrow \infty} \frac{f\left(t ; \theta_{o}\right)}{f\left(t ; \hat{\theta}_{n}\right)} d F\left(t ; \theta_{o}\right)($ as $\ln (x)$ is continuous $)$

$=\int_{B_{k}} \ln \frac{f\left(t ; \theta_{o}\right)}{\lim f\left(t ; \hat{\theta}_{n}\right)} d F\left(t ; \theta_{o}\right)$

$\geq \int_{B_{k}} \ln \frac{\substack{n \rightarrow \infty \\ f\left(t ; \theta_{o}\right)}}{f\left(t ; \theta_{*}\right)} d F\left(t ; \theta_{o}\right)$

(by Eq.(5) and (A9)) (8)

$=\int_{B_{k}} H\left(\frac{f\left(t ; \theta_{o}\right)}{f\left(t ; \theta_{*}\right)}\right) \frac{f\left(t ; \theta_{*}\right)}{f\left(t ; \theta_{o}\right)} d F\left(t ; \theta_{o}\right) \quad(H(t)=t \ln t)$

$=\int_{B_{k}} H\left(\frac{f\left(t ; \theta_{o}\right)}{f\left(t ; \theta_{*}\right)}\right) f\left(t ; \theta_{*}\right) d \mu(t) \quad(a s$

$$
\left.d F\left(t ; \theta_{o}\right)=f\left(t ; \theta_{o}\right) d \mu(t)\right)
$$

$\geq \int_{B_{k}}(-1 / e) f\left(t ; \theta_{*}\right) d \mu(t)($ as $t \ln t \geq-1 / e \forall t>0)$

$\geq-1 / e \quad\left(\right.$ as $\left.\int_{\mathcal{B}_{k}} f\left(t ; \theta_{*}\right) d \mu(t) \in[0,1]\right)$.

Finally,

$\geq \underline{\lim }_{n \rightarrow \infty} \int \ln \frac{f\left(t ; \theta_{o}\right)}{f\left(t ; \hat{\theta}_{n}\right)} d \hat{F}_{n}(t)$ (by Eq.(6))

$=\varliminf_{n \rightarrow \infty} \sum_{k \geq 1} \int_{B_{k}} \frac{f\left(t ; \theta_{o}\right)}{f\left(t ; \hat{\theta}_{n}\right)} d \hat{F}_{n}(t)$ (by Eq.(7))

$=\varliminf_{n \rightarrow \infty} \int_{k \geq 1} \int_{B_{k}} \ln \frac{f\left(t ; \theta_{o}\right)}{f\left(t ; \hat{\theta}_{n}\right)} d \hat{F}_{n}(t) d \nu(k)$

( $d \nu$ is the counting measure)

$\geq \int_{k \geq 1} \underline{\lim _{n \rightarrow \infty}} \int_{B_{k}} \ln \frac{f\left(t ; \theta_{o}\right)}{f\left(t ; \hat{\theta}_{n}\right)} d \hat{F}_{n}(t) d \nu(k)$

(by (b) of Corollary 1 and (9))

$\geq \int_{k \geq 1} \int_{B_{k}} \ln \frac{f\left(t ; \theta_{o}\right)}{f\left(t ; \theta_{*}\right)} d F\left(t ; \theta_{o}\right) d \nu(k) \quad$ (by Eq. (8))

$=\int \ln \frac{f\left(t ; \theta_{o}\right)}{f\left(t ; \theta_{*}\right)} d F\left(t ; \theta_{o}\right)$

$=\int \ln \frac{f\left(t ; \theta_{o}\right)}{f\left(t ; \theta_{*}\right)} f\left(t ; \theta_{o}\right) d \mu(t)$

$\geq 0$ (by Lemma 1 ).

That is, $\int \ln \frac{f\left(t ; \theta_{o}\right)}{f\left(t ; \theta_{*}\right)} f\left(t ; \theta_{o}\right) d \mu(t)=0$. It follows that $\int\left|f\left(x ; \theta_{*}\right)-f\left(x ; \theta_{o}\right)\right| d \mu(x)=0$ by the sec- ond statement of Lemma 1. Consequently $\theta_{*}=\theta_{o}$ by (A2). Since $P\left(\Omega_{o}\right)=1$, the MLE $\hat{\theta} \stackrel{a . s}{\rightarrow} \theta_{o}$ and $f\left(x ; \hat{\theta}_{n}\right) \stackrel{\text { a.s. }}{\rightarrow} f\left(x ; \theta_{o}\right) \forall x \in \mathcal{W}$,

Remark 6. In view of Remarks 3 and 4, under assumptions (A1) and (A2), Theorem 1 presents a simple sufficient condition, namely (A9) or (A10) alone, which is much weaker than all similar results in the literature. Notice that in the literature, even though some people show that the sufficient conditions include (A9), it is not known that the sufficient condition can be (A9) alone. We shall show in Theorem 3 that (A10) alone is the NS condition for the MLE being strong consistent. But (A9) is easier to verify than (A10).

\section{Main Results for Random Vectors.}

In this section, assume that $\mathbf{X}$ is a $p \times 1$ random vector. Notice that (A1) says that $\mathbf{X}_{1}, \ldots, \mathbf{X}_{n}$ are i.i.d. observations from $f(\cdot ; \theta), \theta \in \Theta$. Here $\mathbf{X}_{i}$ can be a random variable or a random vector. (A1), (A2) and (A9), as well as Eq. (3), Lemmas 2 and 4 do not need to be revised except replacing $x$ by $\mathbf{x}\left(=\left(x_{1}, \ldots, x_{p}\right)\right)$, etc.

For a better presentation, we shall first extend Theorem 1 to the case $p=2$. Hereafter, write $\mathbf{x}=$ $\left(x_{1}, x_{2}\right)$, etc., denote $\mathbf{x} \geq \mathbf{y}$ if $x_{i} \geq y_{i}, i \in\{1,2\}$; denote $\mathbf{x}>\mathbf{y}$ if $x_{1} \geq y_{1}$ and $x_{2} \geq y_{2}$ with at least one strict inequality. Lemma 3 is a key in the proof of Theorem 1, and it is extended as follows.

Lemma 5. Given each sequence of bivariate cdf's $\left\{F\left(\cdot ; \theta_{n}\right)\right\}_{n \geq 1}$, there exists a convergence subsequence such that $\lim _{j \rightarrow \infty} F\left(\mathbf{x} ; \theta_{n_{j}}\right)=F\left(\mathbf{x} ; \theta_{*}\right) \forall \mathbf{x}$, where $F\left(\mathbf{x} ; \theta_{*}\right) \in \mathcal{F}_{2}$, a collection of all $F$ such that $F(\mathbf{x}) \geq F(\mathbf{y})$ whenever $\mathbf{x} \geq \mathbf{y}, F(-\infty,-\infty)=0$ and $F(\infty, \infty)=1$.

Proof. Given a sequence of cdf's $F\left(\cdot ; \theta_{n}\right)$, $\{F(x, \infty ; \theta)\}_{n \geq 1}$ is a bounded nondecreasing sequence in $x$. Helly's selection theorem ensures that there exists a convergent subsequence. WLOG, we can assume $\left\{F\left(x, \infty ; \theta_{n}\right)\right\}_{n \geq 1}$ converges. Likewise, $F\left(\infty, x ; \theta_{n}\right)$ is a bounded nondecreasing sequence in $x$. Helly's selection theorem ensures that there exists a convergent subsequence. WLOG, we can assume $F\left(\infty, x ; \theta_{n}\right)$ converges too. Moreover, $\left\{F\left(x, x ; \theta_{n}\right)\right\}_{n \geq 1}$ is also a bounded nondecreasing sequence, thus Helly's selection theorem ensures that there exists a convergent subsequence. WLOG, we can further assume $F\left(x, x ; \theta_{n}\right)$ converges again. Denote the limiting functions of $F\left(x, \infty ; \theta_{n}\right)$, $F\left(\infty, x ; \theta_{n}\right)$ and $F\left(x, x ; \theta_{n}\right)$, by $F_{1}, F_{2}$ and $F_{3}$, respectively. Let $Q_{k}$ be the collection of $i / 2^{k}$ th quantiles of $F_{j}$ 's for $i \in\left\{1,2, \ldots, 2^{k}-1\right\}$ and $j \in\{1,2,3\}$. Notice that $Q_{k}$ is a finite set with at most $3\left(2^{k}-1\right)$ elements. For $k=1$, there is a convergent subsequence of $\left\{F\left(\mathbf{x}, \theta_{n}\right)\right\}_{n \geq 1}$ for $\mathbf{x} \in Q_{1}$, denoted by $\left\{F\left(\mathbf{x}, \theta_{1, n}\right)\right\}_{n \geq 1}$. Inductively, for $k \geq 2$, there is 
a convergent subsequence of $\left\{F\left(\mathbf{x}, \theta_{k-1, n}\right)\right\}_{n \geq 1}$ for $\mathbf{x} \in \cup_{j=1}^{k} Q_{j}$, denoted by $\left\{F\left(\mathbf{x}, \theta_{k, n}\right)\right\}_{n \geq 1}$. Then the subsequence $\left\{F\left(\mathbf{x}, \theta_{n, n}\right)\right\}_{n \geq 1}$ converges $\forall \mathbf{x} \in$ $\cup_{k \geq 1} Q_{k}$. WLOG, we can assume $F\left(\mathbf{x}, \theta_{n}\right)$ converges for $\mathbf{x} \in \cup_{k \geq 1} Q_{k}$.

We now show that $F\left(\mathbf{x} ; \theta_{n}\right)$ converges pointwisely. By the previous construction, it suffices to show that $F\left(\mathbf{x} ; \theta_{n}\right)$ converges if $\mathbf{x} \notin \cup_{j \geq 1} Q_{j}$. In the latter case, given $\epsilon>0, \exists k>0$ and $\exists \mathbf{z}, \mathbf{y} \in \cup_{j} Q_{j}$ satisfying $\mathbf{z}<\mathbf{x}<\mathbf{y}$ such that

$$
\left\{\begin{array}{l}
F\left(\mathbf{y} ; \theta_{n}\right)-F\left(\mathbf{z} ; \theta_{n}\right) \leq \epsilon, \\
\left|F\left(\mathbf{y} ; \theta_{m}\right)-F\left(\mathbf{y} ; \theta_{n}\right)\right| \leq \epsilon, \\
F\left(\mathbf{z} ; \theta_{m}\right)-F\left(\mathbf{z} ; \theta_{n}\right) \mid \leq \epsilon,
\end{array}\right.
$$

whenever $n, m \geq k$. It follows that

$$
\begin{aligned}
& \left|F\left(\mathbf{x} ; \theta_{n}\right)-\bar{F}\left(\mathbf{x} ; \theta_{m}\right)\right| \\
\leq & \mid F\left(\mathbf{y} ; \theta_{m}\right)-F\left(\mathbf{y} ; \theta_{m}\right)+F\left(\mathbf{y} ; \theta_{n}\right)-F\left(\mathbf{y} ; \theta_{n}\right) \\
& +F\left(\mathbf{x} ; \theta_{n}\right)-F\left(\mathbf{x} ; \theta_{m}\right)+F\left(\mathbf{z} ; \theta_{m}\right)-F\left(\mathbf{z} ; \theta_{m}\right) \mid \\
= & \mid F\left(\mathbf{y} ; \theta_{m}\right)-F\left(\mathbf{z} ; \theta_{m}\right)+F\left(\mathbf{y} ; \theta_{n}\right)-F\left(\mathbf{y} ; \theta_{m}\right) \\
& +F\left(\mathbf{x} ; \theta_{n}\right)-F\left(\mathbf{y} ; \theta_{n}\right)+F\left(\mathbf{z} ; \theta_{m}\right)-F\left(\mathbf{x} ; \theta_{m}\right) \mid \\
\leq & \left|F\left(\mathbf{y} ; \theta_{m}\right)-F\left(\mathbf{z} ; \theta_{m}\right)\right|+\left|F\left(\mathbf{y} ; \theta_{n}\right)-F\left(\mathbf{y} ; \theta_{m}\right)\right| \\
& +\left|F\left(\mathbf{x} ; \theta_{n}\right)-F\left(\mathbf{y} ; \theta_{n}\right)\right|+\left|F\left(\mathbf{z} ; \theta_{m}\right)-F\left(\mathbf{x} ; \theta_{m}\right)\right| \\
\leq & \left|F\left(\mathbf{y} ; \theta_{m}\right)-F\left(\mathbf{z} ; \theta_{m}\right)\right|+\left|F\left(\mathbf{y} ; \theta_{n}\right)-F\left(\mathbf{y} ; \theta_{m}\right)\right| \\
& +2\left|F\left(\mathbf{z} ; \theta_{m}\right)-F\left(\mathbf{y} ; \theta_{m}\right)\right|(\text { as } \mathbf{z}<\mathbf{x}<\mathbf{y})
\end{aligned}
$$

$\leq 4 \epsilon$, whenever $n, m \geq k$ by Eq.(10).

Consequently, $F\left(\mathbf{x} ; \theta_{k}\right)$ converges pointwisely. ㅁ

Theorem 2. Suppose that (A1) and (A2) hold and $\mathbf{X}_{i}$ 's in (A1) are 2 -dimensional random vector. Then the MLE $\hat{\theta}$ is consistent if (A9) or (A10) holds.

Proof. Let $\hat{F}_{n}$ be the empirical distribution function (edf) based on $\mathbf{X}_{i}$ 's and $\Omega_{o}$ the subset of the sample space $\Omega$ such that $\hat{F}_{n}(\cdot) \rightarrow F\left(\cdot ; \theta_{o}\right)$, where $F\left(\cdot ; \theta_{o}\right)$ is the true cdf of $\mathbf{X}$. Under assumptions (A1) and (A2), $P\left(\Omega_{o}\right)=1$. For each $\omega \in \Omega_{o}$, let $\theta_{*}$ be a limiting point of $\hat{\theta}_{n}(\omega)$, where $\theta_{*} \in \bar{\Theta}$. Then the MLE $\hat{\theta}_{n}$ is consistent iff $\theta_{*}=\theta_{o}$, Thus the theorem is proved once we prove $\theta_{*}=\theta_{o}$, which is done next.

Hereafter, fixed $\omega \in \Omega_{0}$. Then there exists a convergent subsequence of $\left\{F\left(\cdot ; \hat{\theta}_{n}\right)\right\}_{n \geq 1}$ by Lemma 5 . By taking a convergence subsequences of $\left\{\hat{\theta}_{n}\right\}_{n \geq 1}$ and $\left\{F\left(\cdot ; \hat{\theta}_{n}\right)\right\}_{n \geq 1}$, WLOG, we can assume $\hat{\theta}_{n} \rightarrow \theta_{*}$ and $\hat{F}\left(\cdot, \hat{\theta}_{n}\right)$ converges to $F\left(\cdot ; \theta_{*}\right)$ pointwisely, where $F\left(\cdot ; \theta_{*}\right) \in \mathcal{F}_{2}$. Thus either (A10) yields Eq.(5), or both (A9) and Lemma 4 yield Eq.(4) with $\theta_{n}=\hat{\theta}_{n}$, that is,

$$
\varlimsup_{n \rightarrow \infty} f\left(\mathbf{x} ; \hat{\theta}_{n}\right) \leq f\left(\mathbf{x} ; \theta_{*}\right), \mathbf{x} \in \mathcal{W},
$$

where $\int \mathbf{1}(\mathbf{x} \notin \mathcal{W}) d \mu(\mathbf{x})=0$ (see Eq.(5))

The normalized log-likelihood is $\sum_{i=1}^{n} \ln f\left(\mathbf{X}_{i} ; \theta\right) / n$. Let $H(t)=t \ln t$. We have

$$
\begin{array}{r}
0 \geq \\
\quad \frac{1}{n} \sum_{i=1}^{n} \ln \frac{f\left(\mathbf{X}_{i} ; \theta_{o}\right)}{f\left(\mathbf{X}_{i} ; \hat{\theta}_{n}\right)}=\int \ln \frac{f\left(\mathbf{t} ; \theta_{o}\right)}{f\left(\mathbf{t} ; \hat{\theta}_{n}\right)} d \hat{F}_{n}(\mathbf{t}) \\
\quad=\int H\left(\frac{f\left(\mathbf{t} ; \theta_{o}\right)}{f\left(\mathbf{t} ; \hat{\theta}_{n}\right)}\right) \frac{f\left(\mathbf{t} ; \hat{\theta}_{n}\right)}{f\left(\mathbf{t} ; \theta_{o}\right)} d \hat{F}_{n}(\mathbf{t}) \quad(\operatorname{see}(6)) .
\end{array}
$$

The rest of the proof is skipped, as it is identical to the proof of Theorem 1 after Eq.(6), provided that $x$ is replaced by $\mathbf{x}$. $\square$

Remark 7. The extension of Theorem 1 to the case $p>1$ can be done through a mathematical induction on $p$. The proof of Theorem 1 can be viewed as the step $p=1$ in the mathematical induction. The proof of Theorem 2 can be viewed as the simple version of step $p+1$. For simplicity, we ignore the details.

\section{Direct Applications.}

We first consider the case of a random vector specified in the next example.

Example 3. Let $f_{\mathbf{X}}(\mathbf{x} ; \theta, \alpha, r)=\prod_{i=1}^{3} f_{i}\left(\frac{x_{i}-\alpha}{\theta}\right)$, be the df of $\mathbf{X}=\left(X_{1}, X_{2}, X_{3}\right)$, where $f_{1}(x)=\frac{(r-1) \mathbf{1}(x>e)}{(\ln x)^{r} x}, f_{2}(x)=\frac{(r-1) \mathbf{1}(x \in(0,1 / e))}{(\ln x)^{r} x}$ and $f_{3}(x)=\frac{\mathbf{1}(x>e)+\mathbf{1}(x \in(0,1 / e))}{2(\ln x)^{r} x}, r>1, \theta>0$. Notice that $f_{1}, f_{2}$ and $f_{3}$ are the examples corresponding to Cases 2, 3 and 4 (mentioned in Section 1), respectively. Then the MLE of the parameter $(\theta, \alpha, r)$ based on i.i.d. observations from $f_{\mathbf{X}}$ is consistent. It suffices to show that (A2) and (A9) holds.

$\vdash$ : (A2) holds, i.e., $(\theta, \alpha, r)=\left(\theta_{o}, \alpha_{o}, r_{o}\right)$ if

$$
F(\mathbf{x} ; \theta, \alpha, r)=F\left(\mathbf{x} ; \theta_{o}, \alpha_{o}, r_{o}\right) \forall \mathbf{x} \text {. }
$$

By taking derivative, it is easy to check that $\int \frac{r-1}{x(\ln x)^{r}} d x=-\operatorname{sign}(\ln x)|\ln x|^{1-r}+c$, where $c$ is a constant. Then

$$
\begin{aligned}
& F(\mathbf{x} ; \theta, \alpha, r)=\mathbf{1}\left(x_{1}>\alpha+e \theta\right)\left[1-\left(\ln \frac{x_{1}-\alpha}{\theta}\right)^{1-r}\right] \\
& \quad \times \mathbf{1}\left(x_{2} \in(\alpha, \alpha+\theta / e)\right)\left|\ln \frac{x_{2}-\alpha}{\theta}\right|^{1-r} \\
& \quad \times\left\{\mathbf{1}\left(x_{3} \in(\alpha, \alpha+\theta / e)\right) /\left|\ln \frac{x_{3}-\alpha}{\theta}\right|\right. \\
& \left.\quad+\mathbf{1}\left(x_{3}>\alpha+e \theta\right)\left[1-1 /\left(\ln \frac{x_{3}-\alpha}{\theta}\right)\right]\right\} / 2 .
\end{aligned}
$$

If $(\theta, \alpha, r) \neq\left(\theta_{o}, \alpha_{o}, r_{o}\right), \theta, \theta_{o}>0$, and $r, r_{o}>1$, due to symmetry, it suffices to consider these 4 cases:
(a) $\alpha<\alpha_{o}$,
(b) $\alpha+\theta / e<\alpha_{o}+\theta_{o} / e$,
(c) $\alpha+\theta e<\alpha_{o}+\theta_{o} e$,
(d) $r<r_{o}$.

We shall show that if $F(\mathbf{x} ; \theta, \alpha, r)=F\left(\mathbf{x} ; \theta_{o}, \alpha_{o}, r_{o}\right)$ $\forall \mathbf{x}$ then none of the 4 cases is possible.

In Case (a), $\exists x_{2} \in\left(\alpha, \alpha_{o} \wedge(\alpha+\theta / e)\right)$ and $x_{1}=x_{3} \approx \infty$ such that

$F(\mathbf{x} ; \theta, \alpha, r)=\mathbf{1}\left(x_{2} \in(\alpha, \alpha+\theta / 3)\right)\left|\ln \frac{x_{2}-\alpha}{\theta}\right|^{1-r}$ $>0=F\left(\mathbf{x} ; \theta_{o}, \alpha_{o}, r_{o}\right)$, by Eq.(11). Thus Case (a) is impossible.

In Case (b), $\exists x_{2} \in\left((\alpha+\theta / e) \vee \alpha_{o}, \alpha_{o}+\theta_{o} / e\right)$ and $x_{1}=x_{3} \approx \infty$ such that $F(\mathbf{x} ; \theta, \alpha, r)=0$

$<\mathbf{1}\left(x_{2} \in\left(\alpha_{o}, \alpha_{o}+\theta_{o} / e\right)\right)\left|\ln \frac{x_{2}-\alpha_{o}}{\theta_{o}}\right|^{1-r_{o}}$

$=F\left(\mathbf{x} ; \theta_{o}, \alpha_{o}, r_{o}\right)$ by Eq.(11). Thus Case (b) is impossible.

In Case (c), $\exists x_{1} \in\left(\alpha+\theta e, \alpha_{o}+\theta_{o} e\right)$ and $x_{2}=$ $x_{3} \approx \infty$ such that

$\left.F(\mathbf{x} ; \theta, \alpha, r)=\mathbf{1}\left(x_{1}>\alpha+\theta e\right)\right)\left[1-\left(\ln \frac{x_{1}-\alpha}{\theta}\right)^{1-r}\right]$ $>0=F\left(\mathbf{x} ; \theta_{o}, \alpha_{o}, r_{o}\right)$. Thus Case (c) is impossible. 
The previous discussion implies that if $F(\mathbf{x} ; \theta, \alpha, r)=F\left(\mathbf{x} ; \theta_{o}, \alpha_{o}, r_{o}\right) \forall \mathbf{x}$ then $(\theta, \alpha)=$ $\left(\theta_{o}, \alpha_{o}\right)$ and thus $F(\mathbf{x} ; \theta, \alpha, r)=F\left(\mathbf{x} ; \theta, \alpha, r_{o}\right) \forall$ $\mathbf{x}$. The latter equality together with Eq.(11) and $x_{1}=x_{3}=\infty$ further implies

$\mathbf{1}\left(x_{2} \in(\alpha, \alpha+\theta / e)\right)\left|\ln \frac{x_{2}-\alpha}{\theta}\right|^{1-r}$

$=\mathbf{1}\left(x_{2} \in(\alpha, \alpha+\theta / e)\right)\left|\ln \frac{x_{2}-\alpha}{\theta}\right|^{1-r_{o}}$ for all $x_{2}$.

$=>1=\left|\ln \frac{x_{2}-\alpha}{\theta}\right|^{r_{o}-r}$ if $\left.x_{2} \in(\alpha, \alpha+\theta / e)\right)$.

Letting $x_{2} \downarrow \alpha$, the last equation yields $1 \rightarrow \infty$ which is a contradiction. It implies that $r=r_{o}$. Thus (A2) holds.

Finally, $f_{\mathbf{X}}(\mathbf{x} ; \theta, \alpha, r)$ is continuous in $(\theta, \alpha, r) \forall$ $\mathbf{x} \notin B$, where $\mathbf{x} \in B$ implies that either $x_{1}=\alpha+\theta e$ or $x_{2} \in\{\alpha, \alpha+\theta / e\}$ or $x_{3} \in\{\alpha, \alpha+\theta / e, \alpha+\theta e\}$. Furthermore, $\int \mathbf{1}(\mathbf{x} \in B) d \mathbf{x}=0$. Thus (A9) holds. $\square$ Example 4. Suppose that the survival time $Y \sim U(0, \theta), \theta \in \Theta=\mathcal{R}$. The censoring time $C \sim \operatorname{Exp}(1) . \quad Y$ and $C$ are independent. The observable random vector is $\mathbf{X}=(M, \delta)$, where $M=Y \wedge C$ and $\delta=\mathbf{1}(Y \leq C$. Then $f(m, \delta ; \theta)=\frac{\mathbf{1}(m \in[0, \theta])}{\theta}(\theta-m)^{1-\delta}$. Let $\left(M_{1}, \delta_{1}\right)$, $\ldots,\left(M_{n}, \delta_{n}\right)$ be i.i.d. observations. It can be shown that the MLE of $\theta$ has no closed form solution. It is easy to verify that (A4), (A5) and (A6) do not hold. However, it is easy to show that (A9) holds with $\mathcal{W}=\{0, y\} \times\{0,1\} \forall y>0$. Thus the MLE of $\theta$ is strong consistent by Theorem 2 .

It seems that Theorem 2 is just for non-regression data, however it can also be applied to the regression data. For instance, the common regression model is the linear regression model, which can be specified by $\mathbf{Y}=\beta^{\prime} \mathbf{Z}+\mathbf{W}$, where $\mathbf{Y}$ is a $k$-dimensional response vector, $\mathbf{Z}$ is a $p$-dimensional covariate vector which may take value zero, and $E(W)$ may not exist. The conditional df of $\mathbf{Y}$, given $\mathbf{Z}=\mathbf{z}$, is

$$
f_{\mathbf{y} \mid \mathbf{z}}(\mathbf{y} \mid \mathbf{z})=f_{\mathbf{W}}\left(\mathbf{y}-\beta^{\prime} \mathbf{z} ; \gamma\right) \text {, where } \beta \text { is a }
$$

$k \times p$ dimensional matrix, $\beta$ and $\gamma$ are parameters. The marginal df of $\mathbf{Z}$ is $f_{\mathbf{Z}}(\mathbf{z})$, which does not depend on $(\beta, \gamma)$. Then the joint df of $\mathbf{X}=\left(\mathbf{Y}^{\prime}, \mathbf{Z}^{\prime}\right)^{\prime}$ becomes

$$
f_{\mathbf{X}}(\mathbf{x} ; \theta)=f_{\mathbf{W}}\left(\mathbf{y}-\beta^{\prime} \mathbf{z} ; \gamma\right) f_{\mathbf{z}}(\mathbf{z}), \text { where }
$$

$\theta=(\beta, \gamma)$ and $\mathbf{x}^{\prime}=\left(\mathbf{y}^{\prime}, \mathbf{z}^{\prime}\right)$. For a random sample from the df $f_{\mathbf{X}}(\cdot ; \theta)$, one can apply Theorem 2 to prove the consistency of the MLE, such as the next example. Example 5. Suppose that the df of $\mathbf{X}$ is as in Eq.(12), where $W=\left(W_{1}, W_{2}\right)^{\prime}, W_{1}$ and $W_{2}$ are independent, $f_{W_{i}}(t ; \gamma)=\frac{\gamma_{i}-1}{t(\ln t)^{\gamma_{i}}} \mathbf{1}(t>e)\left(\gamma_{i}>1\right)$. By Eq.(12),

$f_{\mathbf{X}}(\mathbf{x} ; \theta)$

$=f_{W}\left(x_{1}-\sum_{j=1}^{p} \beta_{1 j} z_{j}, \gamma_{1}\right) f_{W}\left(x_{2}-\sum_{j=1}^{p} \beta_{2 j} z_{j}, \gamma_{2}\right) f_{\mathbf{Z}}(\mathbf{z})$,

where $\theta=\left(\gamma_{1}, \gamma_{2}, \beta\right)$, and $\beta$ is a $2 \times p$ matrix. The MLE of $\theta$ can be derived by a numerical method, but the consistency can be proved easily by Theorem 2 . In order to prove its consistency, it is suffices to show

$\varlimsup_{n \rightarrow \infty} f_{\mathbf{X}}\left(\mathbf{x} ; \theta_{n}\right) \leq f_{\mathbf{X}}\left(\mathbf{x} ; \theta_{*}\right)$, whenever $\theta_{n}, \theta_{*} \in \Theta$, and $\theta_{n} \rightarrow \theta_{*}$. Notice that $f_{W_{i}}\left(t_{i}-\sum_{j=1}^{p} \beta_{i j} z_{j} ; \gamma_{i}\right)=$ $\frac{\left(\gamma_{i}-1\right) \mathbf{1}\left(\left(t_{i}-\sum_{j=1}^{p} \beta_{i j} z_{j}\right)>e\right)}{\left(t_{i}-\sum_{j=1}^{p} \beta_{i j} z_{j}\right)\left(\ln \left(t_{i}-\sum_{j=1}^{p} \beta_{i j} z_{j}\right)\right)^{\gamma_{i}}}$, which is continuous in $\theta \in \Theta$ a.e. in $\mathbf{x} \notin B_{\theta}$, where $\mathcal{B}_{\theta}=\left\{\left(t_{1}, t_{2}, z_{1}, \ldots, z_{p}\right): t_{i}-\sum_{j=1}^{p} \beta_{i j} z_{j}=e\right\}$ and $\int_{B_{\theta}} 1 d \mathbf{x}=0$. Thus $f_{\mathbf{x}}(\mathbf{x} ; \theta)$ is continuous a.e. in $\mathbf{x}$. Consequently, (A9) holds and thus the MLE is consistent.

One may modify Theorem 2 for the uncertain MLE under the uncertain regression analysis.

\section{Concluding Remark.}

In this paper, assuming (A1) and (A2), it is shown that (A9) is the only sufficient condition needed for the MLE $\hat{\theta}$ being consistent. In almost all natural parametric distribution families, (A9) is valid and is easy to verified, thus Theorems 1 and 2 confirm our belief that the MLE is consistent in almost all practical cases. Is (A9) also the sufficient condition? See the next example.

Example 6. Let $\mathcal{K}=\{1,2,3, \ldots\}$ and the density be $f= \begin{cases}\mathbf{1}(x \in(0,2]) / 2 & \text { if } \theta=0 \\ \frac{k+1}{k} \exp (-x(k+1) / k) & \text { if } \theta=1 / k, k \in \mathcal{K},\end{cases}$ where $\Theta=\{0\} \cup\{1 / k: k \in \mathcal{K}\}$. Then $\hat{\theta}_{n}$ is consistent, (A10) holds but not (A9) and Eq. (1).

The proof is given in a technical report (Yu [15]). Theorem 3. Suppose that $\mathbf{X}$ is a random vector, (A1) and (A2) hold, $\theta_{o} \in \Theta$ and $\hat{\theta}_{n}$ is the MLE of $\theta_{o}$. Then statement (A10) holds iff statement (A11) holds.

(A11) $\hat{\theta}_{n}(\omega) \rightarrow \theta_{o}$ and $f\left(x ; \hat{\theta}_{n}(\omega)\right) \rightarrow f\left(x ; \theta_{o}\right) \forall$ $x \in \mathcal{W}$ and $\omega \in \Omega_{o}$, where

$\int \mathbf{1}(x \notin \mathcal{W}) d \mu(x)=0$ and $\Omega_{0}$ is a subset of the sample space satisfying $P\left(\Omega_{o}\right)=1$.

Proof. By Theorems 1 and 2, (A10) implies (A11). On the other hand, letting $\theta_{*}=\theta_{o}$ in (A11), it is trivially true that (A11) yields (A10). ㅁ

Remark 8. Theorem 3 actually presents the NS condition for the MLE $\hat{\theta}_{n}$ being strongly consistent, though the consistency is referred to both $\hat{\theta}_{n}$ and $f\left(\cdot ; \hat{\theta}_{n}\right)$.

\section{References:}

[1] Berlinet, A., Liese, F. and Vajda, I., Necessary and sufficient conditions for consistency of Mestimates in regression models with general errors, Journal of Statistical Planning and Inference, Vol.89, No.1-2, 2000, pp. 243-267.

[2] Bickel, P.J. and Doksum, K.A., Mathematical Statistics, Holden-Day, Oakland, 1997. 
[3] Casella, G. and Berger, R., Statistical inference 2nd Ed., Duxbury, NY, 2001.

[4] Derksen H. and Makam V., Maximum Likelihood Estimation for Matrix Normal Models via Quiver Representations SIAM J. Appl. Algebra Geometry, Vol.5, 2021, pp.338-365.

[5] Ferguson, T.S., A course in large sample theory, hapman \& Hall, NY, 1996.

[6] Kullback, S. and Leibler, R. A., On information and sufficiency. Ann. Math. Stat., Vol.22, 1951, pp.79-86.

[7] Lehmann, E.L. and Casella, G., Theory of Point estimation 2nd edition, Springer-Verlag, NY, 1998.

[8] Lio, W. and Liu, B., Uncertain Maximum Likelihood Estimation with Application to Uncertain Regression Analysis Soft Computing, Vol. 24, 2020, pp. 9351-9360.

[9] Rossi, R. J., Mathematical Statistics : An Introduction to Likelihood Based Inference, pp. 227. John Wiley \& Sons, NY, 2018.

[10] Royden, H.L., Real analysis, Macmillan, NY, 1968.

[11] Rudin, W., Principles of mathematical analysis, McGraw-Hill, NY, 1976.

[12] Stuart, A. Ord, J.K., and Arnold, S., Advanced Theory of Statistics, Vol. 2A: Classical Inference

\section{Creative Commons Attribution \\ License 4.0 (Attribution 4.0 \\ International, CC BY 4.0)}

This article is published under the terms of the Creative Commons Attribution License 4.0

and the Linear Model, 6th edition. Oxford University Press, London, 1999.

[13] Van der Vaart, A. W., Asymptotic Statistics, Cambridge University Press, 1998.

[14] Yu, Q.Q., Consistency Of The Semi-parametric MLE Under The Cox Model With RightCensored Data, The Open Mathematics, Statistics and Probability Journal Vol.10 2020, pp.21-27.

[15] Yu, Q.Q., Technical Report on "The necessary and sufficient condition for consistency of the MLE", 2021. http://people.math.binghamton.edu/qyu/ftp/cmle.pdf

[16] Zhang, J., Consistency of MLE, LSE and Mestimation under mild conditions. Statistical $\mathrm{Pa}$ pers Vol.61, 2017, pp.189-199.

https://creativecommons.org/licenses/by/4.0/deed.en US 